\title{
Classical relativistic systems of charged particles in the front form of dynamics and the Liouville equation*
}

\author{
A.Nazarenko, V.Tretyak \\ Institute for Condensed Matter Physics \\ of the National Academy of Sciences of Ukraine, \\ 1 Svientsitskii Str., 79011 Lviv, Ukraine
}

Received December 20, 1999

\begin{abstract}
Classical relativistic system of point particles coupled with an electromagnetic field is considered in the three-dimensional representation. The gauge freedom connected with the chronometrical invariance of the four-dimensional description is reduced by use of the geometrical concept of the forms of relativistic dynamics. The remainder gauge degrees of freedom of the electromagnetic potential are analysed within the framework of Dirac's constrained Hamiltonian mechanics in the front form of dynamics. The results are applied to the problems of relativistic statistical mechanics. Based on the corresponding Liouville equation the classical partition function of the system is written down in a gauge-invariant manner and an integration over field variables is performed.
\end{abstract}

Key words: classical relativistic mechanics, forms of relativistic dynamics, relativistic statistical mechanics, charged particles, Liouville equation

PACS: 03.30. $+p, 05.20 .-y$

\section{Introduction}

All fundamental interactions in physics have got gauge nature and demand the use of singular Lagrangians. An adequate method for dealing with such systems was developed by Dirac (see [1-3]). His approach, i.e., constrained Hamiltonian mechanics, has been elaborated in many directions $[4,5]$ and usefully applied to the problems of quantum field theory (see, e.g., [6,7]). But an application of this technique to the relativistic statistical mechanics $[8,9]$ seems rather obscure, although, for example, the thermo field formulation of the condensed matter theory [10] admits a variational approach with the same gauge structure as in quantum electrodynamics and in related theories. We only note the paper [11], where the use of Faddeev' measure

${ }^{*}$ This is an invited paper to the special issue on the problems of thermofield dynamics. 
[6] was suggested for the construction of the relativistic partition function of directly interacting particle system described by means of the constrained Hamiltonian formalism.

The present paper is concerned with the classical relativistic system of point charges coupled with an electromagnetic field. The first attempt to analyse the constraint contents of such a system was made by Dirac [12]. The corresponding action functional in the four-dimensional representation has got two kinds of the gauge freedom. The first is connected with arbitrariness in the parametrization of particle world lines (chronometrical invariance); the second is generated by the proper gauge transformations of electromagnetic potentials. We reduce the gauge freedom of the first kind by means of the farther great Dirac's invention, namely, the concept of the forms of relativistic dynamics [13]. Here we use the notion of the form of dynamics as denoting the description of relativistic system, which corresponds to a given global simultaneity relation defined by means of some foliation of the Minkowski space by space-time or isotropic hypersurfaces. Moreover, we consider only the case when the corresponding simultaneity relation is independent of particle or field configurations (cf. [14]). Using Dirac's constraint formalism, we try to isolate the gauge degrees of freedom and to formulate the statistical description of the system in a gauge-invariant manner.

The general features of the formalism were presented in [15] within the instant form of dynamics. It will be our purpose in the present paper to explore the further possibilities connected with the other forms of relativistic dynamics; in particular, we shall consider the front form description of the charged particle system.

The paper is organised as follows. In section 2 we will first establish the structure of Hamiltonian description of the system of particles plus field in an arbitrary form of relativistic dynamics. Our results are then applied to the front form of dynamics. In section 3 we perform the analysis of the corresponding constraints. Section 4 contains the elimination of the gauge degrees of freedom by suitable canonical transformation. Sections 5 and 6 are devoted to the application in relativistic statistical mechanics. We formulate a Liouville equation for distribution function in the front form of dynamics and write down its equilibrium solution corresponding to classical Gibbs ensemble accounting particle and field degrees of freedom. Peculiarity of the front form of dynamics allows us to perform an integration over field variables in a relativistic partition function. Some calculations of a purely technical nature are collected in appendices. 


\section{Charged particle system in an arbitrary form of dynamics}

We shall consider a system of $N$ charged particles, which is described by their (time-like) world lines in the Minkowski space-time ${ }^{1}$

$$
\gamma_{a}: \mathbb{R} \mapsto \mathbb{M}_{4}, \quad \tau \mapsto x_{a}^{\mu}(\tau)
$$

An interaction between charges is assumed to be mediated by an electromagnetic field $F=d A$ with the electromagnetic potential 1-form

$$
\begin{gathered}
A=\tilde{A}_{\mu}(x) \mathrm{d} x^{\mu} \\
F=\frac{1}{2} \tilde{F}_{\mu \nu}(x) \mathrm{d} x^{\mu} \wedge \mathrm{d} x^{\nu}, \quad \tilde{F}_{\mu \nu}(x)=\partial_{\mu} \tilde{A}_{\nu}-\partial_{\nu} \tilde{A}_{\mu},
\end{gathered}
$$

$\partial_{\nu} \equiv \partial / \partial x^{\nu}$. The dynamical properties of such a system are completely determined by the following action functional [16-18]

$$
S=\sum_{a=1}^{N} \int \mathrm{d} \tau_{a}\left\{-m_{a} \sqrt{-u_{a}^{2}\left(\tau_{a}\right)}+e_{a} u_{a}^{\nu}\left(\tau_{a}\right) \tilde{A}_{\nu}\left[x_{a}\left(\tau_{a}\right)\right]\right\}-\frac{1}{16 \pi} \int \mathrm{d}^{4} x \tilde{F}_{\mu \nu}(x) \tilde{F}^{\mu \nu}(x),
$$

where $m_{a}$ and $e_{a}$ denote the mass and the charge of particle $a$, respectively, $u_{a}^{\mu}\left(\tau_{a}\right)=$ $\mathrm{d} x_{a}^{\mu}\left(\tau_{a}\right) / \mathrm{d} \tau_{a}$. We are interested in constructing the Hamiltonian description of the system in a given form of relativistic dynamics. The particle and field degrees of freedom will be treated on equal level.

The form of relativistic dynamics in its geometrical definition is specified by the foliation $\Sigma=\left\{\Sigma_{t} \mid t \in \mathbb{R}\right\}$ of the Minkowski space-time with space-like or isotropic hypersurfaces

$$
\begin{gathered}
\Sigma_{t}=\left\{x \in \mathbb{M}_{4} \mid \sigma(x)=t\right\} ; \\
\eta^{\mu \nu}\left(\partial_{\mu} \sigma\right)\left(\partial_{\nu} \sigma\right) \leqslant 0 .
\end{gathered}
$$

(see [19-21]). As it follows from condition (2.6), $\partial_{0} \sigma \neq 0$ and therefore the hypersurface equation $(2.5)$ can be solved with respect to $x^{0}$ in the form:

$$
x^{0}=\varphi(t, \mathbf{x}), \quad \mathbf{x}=\left(x^{1}, x^{2}, x^{3}\right) .
$$

For definiteness we put

$$
\partial_{0} \sigma>0 \quad \text { and } \quad \frac{\partial \varphi(t, \mathbf{x})}{\partial t} \equiv \varphi_{t}(t, \mathbf{x})>0 .
$$

Making use of the identities

$$
\left.\partial_{0} \sigma\right|_{\Sigma_{t}}=\varphi_{t}^{-1},\left.\quad \partial_{i} \sigma\right|_{\Sigma_{t}}=-\varphi_{i} \varphi_{t}^{-1}, \quad \varphi_{i} \equiv \partial \varphi / \partial x^{i}
$$

\footnotetext{
${ }^{1}$ The Minkowski space-time $\mathbb{M}_{4}$ is endowed with a metric $\left\|\eta_{\mu \nu}\right\|=\operatorname{diag}(-1,1,1,1)$. The Greek indices $\mu, \nu, \ldots$ run from 0 to 3 ; the Latin indices from the middle of alphabet, $i, j, k, \ldots$, run from 1 to 3 and both types of indices are subject of the summation convention. The Latin indices from the beginning of alphabet, $a, b$, label the particles and run from 1 to $N$. The sum over such indices is pointed explicitly. The velocity of light and the Planck constant $\hbar$ are equal to unity.
} 
it is easy to see that condition (2.6) implies the following inequality for the function $\varphi$ :

$$
\varphi^{2} \leqslant 1, \quad \boldsymbol{\varphi}=\left(\frac{\partial \varphi}{\partial x^{1}}, \frac{\partial \varphi}{\partial x^{2}}, \frac{\partial \varphi}{\partial x^{3}}\right)
$$

Then one can consider the particle world lines $\gamma_{a}$ as being determined by a set of points $x_{a}(t)=\gamma_{a} \bigcap \Sigma_{t}$ of intersections with the elements of the foliation $\Sigma$. The parametrical equation of the world line in a given form of dynamics is

$$
x^{0}=x_{a}^{0}(t)=\varphi\left(t, \mathbf{x}_{a}(t)\right) \equiv \varphi_{a}, \quad x^{i}=x_{a}^{i}(t) .
$$

The variable $t$ serves as a common evolution parameter of the particle system. Therefore, the choice of the form of dynamics is equivalent to the fixing of the parameters $\tau_{a}=t$ of the particle world lines in the reparametrization-invariant action (2.4).

On the other hand, the foliation $\Sigma$ specifies a certain $3+1$ splitting of the Minkowski space-time $\mathbb{M}_{4} \cong \mathbb{R} \times \Sigma_{0}$, because by the definition of a foliation all hypersurfaces $\Sigma_{t}$ for different $t$ are diffeomorphic to $\Sigma_{0}$. That splitting, $f: \mathbb{M}_{4} \rightarrow$ $\mathbb{R} \times \Sigma_{0}$, is really determined by $(2.7)$ :

$$
f:\left(x^{0}, \mathbf{x}\right) \mapsto(\varphi(t, \mathbf{x}), \mathbf{x}) .
$$

Accounting that the determinant of the transformation (2.12) is

$$
\frac{\partial\left(x^{0}, \mathbf{x}\right)}{\partial(t, \mathbf{x})}=\varphi_{t}(t, \mathbf{x})
$$

one can immediately rewrite the action functional (2.4) into the form

$$
S=\int \mathrm{d} t L
$$

with the Lagrangian function

$$
\begin{gathered}
L=\sum_{a=1}^{N}\left\{-m_{a} \sqrt{\left(D \varphi_{a}\right)^{2}-\mathbf{v}_{a}^{2}}+e_{a}\left[A_{0}\left(t, \mathbf{x}_{a}\right) D \varphi_{a}+v_{a}^{i} A_{i}\left(t, \mathbf{x}_{a}\right)\right]\right\}+\int \mathrm{d}^{3} x \mathcal{L} \\
\mathcal{L}=-\frac{1}{16 \pi} \varphi_{t}(t, \mathbf{x}) F_{\mu \nu} F^{\mu \nu}
\end{gathered}
$$

Here $\mathbf{v}_{a}=\mathrm{d} \mathbf{x}_{a} / \mathrm{d} t, D=\mathrm{d} / \mathrm{d} t=\partial / \partial t+v_{a}^{i} \partial / \partial x_{a}^{i}$ and $A_{\mu}=\tilde{A}_{\mu} \circ f^{-1}, F_{\mu \nu}=\tilde{F}_{\mu \nu} \circ f^{-1}$. The dynamical variables of our variational problem will be the functions $\mathbf{x}_{a}(t)$, $A^{\mu}(t, \mathbf{x})$ and their first order derivatives with respect to the evolution parameter, $\mathbf{v}_{a}(t)$ and $A^{\mu}{ }_{, t}(t, \mathbf{x})$. Using the obvious relations

$$
\partial_{0} \tilde{A}_{\mu}=A_{\mu, t} \varphi_{t}^{-1}, \quad \partial_{i} \tilde{A}_{\mu}=A_{\mu, i}-A_{\mu, t} \varphi_{i} \varphi_{t}^{-1},
$$

we find the following expression for the field Lagrangian density:

$$
\mathcal{L}=\frac{\varphi_{t}}{8 \pi}\left(e_{i} e^{i}-h_{i} h^{i}\right)
$$


where

$$
e_{i}=-A_{0, i}+\left(A_{i, t}+A_{0, t} \varphi_{i}\right) \varphi_{t}^{-1}, \quad h_{i}=\varepsilon_{i j k}\left(A^{k, j}-A^{k}{ }_{t} \varphi^{j} \varphi_{t}^{-1}\right) .
$$

The canonical momenta of our problem are given by

$$
\begin{gathered}
p_{a i}=\frac{\partial L}{\partial v_{a}^{i}}=\frac{m_{a}\left(v_{a i}-\varphi_{a i} D \varphi_{a}\right)}{\sqrt{\left(D \varphi_{a}\right)^{2}-\mathbf{v}_{a}^{2}}}+e_{a}\left[A_{i}\left(t, \mathbf{x}_{a}\right)+A_{0}\left(t, \mathbf{x}_{a}\right) \varphi_{a i}\right], \\
\Pi_{i}(t, \mathbf{x})=\frac{\partial \mathcal{L}}{\partial A^{i}, t}=\frac{1}{4 \pi}\left(e_{i}-\varepsilon_{i j k} \varphi^{j} h^{k}\right), \\
\Pi(t, \mathbf{x})=-\frac{\partial \mathcal{L}}{\partial A_{0, t}}=-\frac{1}{4 \pi} e_{i} \varphi^{i} .
\end{gathered}
$$

The basic Poisson brackets are

$$
\begin{aligned}
& \left\{x_{a}^{i}, p_{b j}\right\}=\delta_{a b} \delta_{j}^{i} \\
& \left\{A_{i}(t, \mathbf{x}), \Pi_{j}(t, \mathbf{y})\right\}=\delta_{i j} \delta^{3}(\mathbf{x}-\mathbf{y}) \\
& \left\{A_{0}(t, \mathbf{x}), \Pi(t, \mathbf{y})\right\}=-\delta^{3}(\mathbf{x}-\mathbf{y})
\end{aligned}
$$

all other brackets vanish. Equations (2.21), (2.22) imply the primary constraint

$$
\xi \equiv \Pi+\Pi^{i} \varphi_{i}=0
$$

In the isotropic forms of dynamics, however, some additional primary constraints may occur. To see that, we rewrite equation (2.21) into the form

$$
\Pi_{i}(t, \mathbf{x})=\frac{1}{4 \pi}\left(\mathfrak{S}_{i}+\beta_{i j} A^{j}{ }_{, t} \varphi_{t}^{-1}+A_{0, t} \varphi_{i} \varphi_{t}^{-1}\right),
$$

where $\mathfrak{S}_{i}$ does not contain the derivatives with respect to the evolution parameter $t$,

$$
\mathfrak{S}_{i}=-A_{0, i}-\varphi^{j} h_{i j} ; \quad h_{i j} \equiv A_{j, i}-A_{i, j}
$$

and

$$
\beta_{i j}=\left(1-\varphi^{2}\right) \delta_{i j}+\varphi_{i} \varphi_{j}
$$

Upon using the identity $\beta_{i j} \varphi^{j}=\varphi_{i}$, one obtains equation

$$
\beta_{i j}\left(A^{j}{ }_{t}+A_{0, t} \varphi^{j}\right)=\varphi_{t}\left(4 \pi \Pi_{i}-\mathfrak{S}_{i}\right) .
$$

The matrix $\beta=\left\|\beta_{i j}\right\|$ has determinant $\operatorname{det} \beta=\left(1-\varphi^{2}\right)^{2}$.

In the case of space-like forms of dynamics $\left(\varphi^{2}<1\right)$ the matrix is nonsingular and possesses an inverse

$$
\gamma_{i j}=\frac{\delta_{i j}-\varphi_{i} \varphi_{j}}{1-\varphi^{2}}, \quad \beta^{i j} \gamma_{j k}=\delta_{k}^{i}
$$

so that equation (2.28) can be solved as

$$
A_{i, t}+A_{0, t} \varphi_{i}=\gamma_{i j} \varphi_{t}\left(4 \pi \Pi^{j}-\mathfrak{S}^{j}\right) .
$$


For isotropic forms of dynamics we have $\beta_{i j}=\varphi_{i} \varphi_{j}$ and (2.28) implies constraints

$$
\xi_{i} \equiv \varepsilon_{i j k} \varphi^{j}\left(4 \pi \Pi^{k}-\mathfrak{S}^{k}\right)=0
$$

Because it holds identically $\varphi^{i} \xi_{i}=0$, really only two additional primary constraints occur.

Next, we consider the canonical Hamiltonian of our system, which is defined as

$$
H_{\mathrm{c}}=\sum_{a=1}^{N} p_{a i} v_{a}^{i}+\int \mathrm{d}^{3} x\left(\Pi^{i} A_{i, t}-\Pi A_{0, t}\right)-L .
$$

The immediate calculations give

$$
H_{\mathrm{c}}=\sum_{a=1}^{N} H_{a}\left(t, \mathbf{x}_{a}, \mathbf{p}_{a}\right)+\int \mathrm{d}^{3} x \mathcal{H}_{\mathrm{c}}
$$

with

$$
H_{a}=\frac{m_{a} \varphi_{a t} D \varphi_{a}}{\sqrt{\left(D \varphi_{a}\right)^{2}-\mathbf{v}_{a}^{2}}}-e_{a} \varphi_{a t} A_{0}\left(t, \mathbf{x}_{a}\right)
$$

and

$$
\mathcal{H}_{\mathrm{c}}=\frac{1}{4 \pi}\left[\frac{1}{2} \varphi_{t}\left(e_{i} e^{i}+h_{i} h^{i}\right)+e^{i} A_{0, i}-\varepsilon_{i j k} A_{, t}^{i} \varphi^{j} h^{k}\right] .
$$

The problem of solution of (2.20) with respect to the particle velocities is analogous to the case of free particles [22]. For completeness we collect the corresponding expressions in Appendix. Replacing into (A.9) $p_{i}$ by

$$
k_{a i} \equiv p_{a i}-e_{a}\left[A_{i}\left(t, \mathbf{x}_{a}\right)+A_{0}\left(t, \mathbf{x}_{a}\right) \varphi_{a i}\right]
$$

yields the one-particle Hamiltonian for the space-like forms of dynamics

$$
H_{a}=\frac{\varphi_{a t}}{1-\varphi_{a}^{2}}\left[\sqrt{\left(1-\varphi_{a}^{2}\right)\left(m_{a}^{2}+\mathbf{k}_{a}^{2}\right)+\left(k_{a}^{i} \varphi_{a i}\right)^{2}}+k_{a}^{i} \varphi_{a i}\right]-e_{a} \varphi_{a t} A_{0}\left(t, \mathbf{x}_{a}\right) .
$$

Similarly, for the isotropic forms of dynamics we obtain from (A.11)

$$
H_{a}=-\frac{1}{2} \varphi_{a t} \frac{m_{a}^{2}+\mathbf{k}_{a}^{2}}{k_{a}^{i} \varphi_{a i}}-e_{a} \varphi_{a t} A_{0}\left(t, \mathbf{x}_{a}\right)
$$

To express the field Hamiltonian (2.35) in the terms of canonical variables we rewrite it into the form

$$
\mathcal{H}_{\mathrm{c}}=\frac{1}{8 \pi}\left\{\varphi_{t}\left[\frac{1}{2} h_{i j} h^{i j}+A_{0, i} A^{0, i}\right]+\varphi_{t}^{-1} \beta_{i j}\left(A_{, t}^{i}+A_{0, t} \varphi^{i}\right)\left(A^{j}{ }_{, t}+A_{0, t} \varphi^{j}\right)\right\} .
$$

Then for the space-like forms of dynamics, taking into account (2.29), (2.30), we obtain

$$
\mathcal{H}_{\mathrm{c}}=\frac{1}{8 \pi} \varphi_{t}\left\{\gamma^{i j}\left(4 \pi \Pi_{i}-\mathfrak{S}_{i}\right)\left(4 \pi \Pi_{j}-\mathfrak{S}_{j}\right)+\frac{1}{2} h_{i j} h^{i j}+A_{0, i} A^{0, i}\right\} .
$$


In the case of isotropic forms of dynamics $\left(\varphi^{2}=1\right)$, when $\beta_{i j}=\varphi_{i} \varphi_{j},(2.28)$ gives

$$
\varphi_{j}\left(A^{j}, t+A_{0, t} \varphi^{j}\right)=\varphi_{t} \varphi_{j}\left(4 \pi \Pi^{j}-\mathfrak{S}^{j}\right),
$$

so that (2.39) takes the form

$$
\mathcal{H}_{\mathrm{c}}=\frac{1}{8 \pi} \varphi_{t}\left\{\left[\varphi^{i}\left(4 \pi \Pi_{i}-\mathfrak{S}_{i}\right)\right]^{2}+\frac{1}{2} h_{i j} h^{i j}+A_{0, i} A^{0, i}\right\} .
$$

Detailed analysis of the Hamiltonian description for the charged particle system in the instant form of dynamics was carried out in [15] with the application to the classical relativistic statistical mechanics. In the following sections we shall be concerned with the front form of dynamics, analyzing additional primary constraints and ensuring the corresponding Liouville equation.

\section{Constraint analysis in the front form of dynamics}

Let us consider the family of the forms of dynamics, which is given by

$$
x^{0}=\tau+\mathbf{n x}, \quad \mathbf{n}^{2}=1 .
$$

According to $(2.24),(2.31)$, the set of primary constraints is

$$
\begin{aligned}
& \xi \equiv \Pi+\Pi^{i} n_{i}=0, \\
& \xi_{i} \equiv \varepsilon_{i j k} n^{j}\left(4 \pi \Pi^{k}+A_{0}{ }^{, k}-n_{l} h^{l k}\right)=0 .
\end{aligned}
$$

It is easy to see that (3.3) is equivalent to

$$
\left(4 \pi \Pi^{k}+A_{0}{ }^{k}\right)_{\perp}-n_{j} h^{j k}=0,
$$

where we define orthogonal and longitudinal projections of an arbitrary 3-vector $f^{k}$ with respect to the vector $n^{k}$ as

$$
f^{k}=f_{\perp}^{k}+f_{\|}^{k}, \quad f_{\|}^{k}=n^{k} n_{l} f^{l}, \quad f_{\perp}^{k}=f^{k}-n^{k} n_{l} f^{l} .
$$

The canonical Hamiltonian of the system is determined by

$$
\begin{aligned}
H_{\mathrm{c}}= & -\sum_{a=1}^{N}\left[\frac{m_{a}^{2}+\mathbf{k}_{a}^{2}}{2 \mathbf{k}_{a} \mathbf{n}}+e_{a} A_{0}\left(t, \mathbf{x}_{a}\right)\right] \\
& +\frac{1}{8 \pi} \int\left\{\left[n^{i}\left(4 \pi \Pi_{i}+A_{0, i}\right)\right]^{2}+\frac{1}{2} h_{i j} h^{i j}+A_{0, i} A^{0, i}\right\} \mathrm{d}^{3} x .
\end{aligned}
$$

Then we get Dirac Hamiltonian which takes into account primary constraints (3.2) and (3.4):

$$
H_{\mathrm{D}}=H_{\mathrm{c}}+\int\left[\lambda\left(\Pi+\Pi^{i} n_{i}\right)+\lambda_{k}\left(\left(4 \pi \Pi^{k}+A_{0}{ }^{, k}\right)_{\perp}-n_{j} h^{j k}\right)\right] \mathrm{d}^{3} x
$$


where $\lambda, \lambda_{k}$ are the Dirac multipliers.

The preservation of the constraint (3.2) in time produces the secondary constraint:

$$
\begin{aligned}
0 & =\left\{\Pi+\Pi^{i} n_{i}, H_{\mathrm{c}}\right\}=-\rho-\partial_{i} \Pi_{\|}^{i}+\frac{\partial_{i}}{4 \pi}\left[\left(A_{0}{ }^{i}\right)_{\perp}-n_{j} h^{j i}\right] \\
& \approx-\Pi_{, i}^{i}-\rho
\end{aligned}
$$

where $\approx$ means "weak equality" in the sense of Dirac and

$$
\rho(t, \mathbf{x})=\sum_{a=1}^{N} e_{a} \delta^{3}\left(\mathbf{x}-\mathbf{x}_{a}(\tau)\right)
$$

is a charge density.

Next, we consider commutation relations between constraints (3.2), (3.4), and (3.8):

$$
\begin{aligned}
& \left\{\Pi+\Pi^{i} n_{i},\left(4 \pi \Pi^{k}+A_{0}{ }^{, k}\right)_{\perp}-n_{j} h^{j k}\right\}=0 \\
& \left\{\Pi^{i}{ }_{, i}+\rho,\left(4 \pi \Pi^{k}+A_{0}{ }^{, k}\right)_{\perp}-n_{j} h^{j k}\right\}=0 \\
& \left\{\left(4 \pi \Pi^{k}(t, \mathbf{x})+A_{0}{ }^{, k}(t, \mathbf{x})\right)_{\perp}-n_{j} h^{j k}(t, \mathbf{x})\right. \\
& \left.\quad\left(4 \pi \Pi^{i}(t, \mathbf{y})+A_{0}{ }^{, i}(t, \mathbf{y})\right)_{\perp}-n_{j} h^{j i}(t, \mathbf{y})\right\}= \\
& =-8 \pi\left(\delta^{k i}-n^{k} n^{i}\right)\left(n^{j} \frac{\partial}{\partial x^{j}}\right) \delta^{3}(\mathbf{x}-\mathbf{y}) \equiv \Omega^{k i}(\mathbf{x}-\mathbf{y}) .
\end{aligned}
$$

We can check directly, that

$$
\left\{\Pi_{, i}^{i}+\rho, H_{\mathrm{c}}\right\}=0
$$

Therefore, the two constraints

$$
\xi \equiv \Pi+\Pi^{i} n_{i}=0, \quad \Gamma \equiv-\Pi^{i}{ }_{i}-\rho \approx 0,
$$

belong to the first class.

Taking into account (3.12), we come to the conclusion that the constraints (3.4) are of the second class. However, there are two independent second class constraints only. Then we can reduce them by means of Dirac bracket:

$$
\begin{aligned}
& \{F, G\}_{\mathrm{D}}=\{F, G\}-\int \mathrm{d}^{3} x \mathrm{~d}^{3} y\left\{F,\left(4 \pi \Pi^{\alpha}(t, \mathbf{x})+A_{0}{ }^{\alpha}(t, \mathbf{x})\right)_{\perp}-n_{i} h^{i \alpha}(t, \mathbf{x})\right\} \\
& \quad \times C_{\alpha \beta}(\mathbf{x}-\mathbf{y})\left\{\left(4 \pi \Pi^{\beta}(t, \mathbf{y})+A_{0}, \beta(t, \mathbf{y})\right)_{\perp}-n_{j} h^{j \beta}(t, \mathbf{y}), G\right\}, \alpha, \beta=1,2,
\end{aligned}
$$

where $C_{\alpha \beta}(\mathbf{x}-\mathbf{y})$ is an inverse matrix to $\Omega^{\alpha \beta}(\mathbf{x}-\mathbf{y})$ :

$$
\int C_{\alpha \gamma}(\mathbf{x}-\mathbf{z}) \Omega^{\gamma \beta}(\mathbf{z}-\mathbf{y}) \mathrm{d}^{3} z=\delta_{\alpha}^{\beta} \delta^{3}(\mathbf{x}-\mathbf{y}) .
$$

In the next chapter we shall consider the elimination of the first class constraints and the formulation of the Hamiltonian description in the terms of independent physical variables. 
Now let us canonically transform the field variables:

$$
\begin{aligned}
& \left(A_{0}, \Pi, A_{i}, \Pi_{i}\right) \mapsto\left(A_{0}, \mathcal{E}, \mathcal{A}_{i}, \mathcal{E}_{i}\right), \\
& \mathcal{E}=\Pi+\Pi^{i} n_{i}, \mathcal{E}_{i}=\Pi_{i}, \mathcal{A}_{i}=A_{i}+A_{0} n_{i} .
\end{aligned}
$$

After the transformation the set of constraints of our system becomes

$$
\begin{aligned}
& \mathcal{E}=0, \Gamma \equiv-\mathcal{E}^{i}{ }_{, i}-\rho \approx 0, \\
& 4 \pi \mathcal{E}_{\perp}^{k}-n_{j}\left(\mathcal{A}^{k, j}-\mathcal{A}^{j, k}\right)=0 .
\end{aligned}
$$

Using (3.19), we can rewrite the canonical Hamiltonian as

$$
\begin{aligned}
H_{\mathrm{c}}= & -\sum_{a=1}^{N}\left[\frac{m_{a}^{2}+\mathbf{k}_{a}^{2}}{2 \mathbf{k}_{a} \mathbf{n}}+e_{a} A_{0}\left(t, \mathbf{x}_{a}\right)\right] \\
& +\int\left\{2 \pi\left(n^{i} E_{i}\right)^{2}-\frac{1}{8 \pi} \mathcal{A}_{i, j}\left(\mathcal{A}^{j, i}-\mathcal{A}^{i, j}\right)-A_{0} \mathcal{E}^{i}{ }_{, i}\right\} \mathrm{d}^{3} x
\end{aligned}
$$

where now $k_{a i}=p_{a i}-e_{a} \mathcal{A}_{i}\left(t, \mathbf{x}_{a}\right)$.

\section{Elimination of the gauge degrees of freedom}

Let us consider the first class constraints. We see immediately that $A_{0}, \mathcal{E}$ are a pair of conjugated gauge canonical variables. Such a second pair is formed by $Q=\Delta^{-1} \mathcal{A}_{, i}^{i}$ and $\Gamma$, where $\Delta=\partial_{i} \partial_{i}, \Delta \Delta^{-1}=1, \Delta_{\mathbf{x}}^{-1} \delta^{3}(\mathbf{x})=\Delta^{-1}(\mathbf{x}) \equiv-1 /(4 \pi|\mathbf{x}|)$.

Therefore, we can separate the gauge degrees of freedom and the gauge-invariant ones by means of the Hodge decomposition (see, e.g., $[23,14]$ ):

$$
\mathcal{A}_{i}=\stackrel{\perp}{\mathcal{A}}_{i}+\partial_{i} Q, \quad \mathcal{E}_{i}=\stackrel{\perp}{\mathcal{E}_{i}}+\partial_{i} \Delta^{-1}(\Gamma+\rho)
$$

where

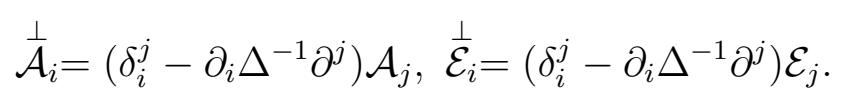

Then we have

$$
\{Q(t, \mathbf{x}), \Gamma(t, \mathbf{y})\}=\delta^{3}(\mathbf{x}-\mathbf{y}),\left\{\stackrel{\perp}{\mathcal{A}}_{i}(t, \mathbf{x}), \stackrel{\perp}{\mathcal{E}}_{j}(t, \mathbf{y})\right\}=\left(\delta_{i j}-\partial^{i} \partial^{j} \Delta^{-1}\right) \delta^{3}(\mathbf{x}-\mathbf{y}) .
$$

Since $\stackrel{\perp}{\mathcal{E}^{i}}, i=0$, we can define $\stackrel{\perp}{\mathcal{E}^{i}}$ as follows

$$
\stackrel{\perp}{\mathcal{E}^{i}}=\left(\delta_{\alpha}^{i}-\delta_{3}^{i} \frac{\partial_{\alpha}}{\partial_{3}}\right) \frac{\tilde{e}^{\alpha}}{\sqrt{4 \pi}}, \quad \tilde{e}^{\alpha}=\sqrt{4 \pi} \mathcal{E}^{\alpha}, \quad \alpha=1,2
$$

Now we have to do a canonical transformation to the new variables $\left(\left(x_{a}^{i}, \tilde{\pi}_{a i}\right)\right.$, $\left.\left(a_{\alpha}, \tilde{e}_{\alpha}\right),(Q, \Gamma),\left(A_{0}, \mathcal{E}\right)\right)$, which is generated by the functional

$$
F=\sum_{a=1}^{N} x_{a}^{i} p_{a i}-\int A_{i}\left[\left(\delta_{\alpha}^{i}-\delta_{3}^{i} \frac{\partial_{\alpha}}{\partial_{3}}\right) \frac{\tilde{e}^{\alpha}}{\sqrt{4 \pi}}+\partial^{i} \Delta^{-1}(\Gamma+\rho)\right] \mathrm{d}^{3} x .
$$


We obtain

$$
\begin{aligned}
& a_{\alpha}=-\frac{\delta F}{\delta e^{\alpha}}=\left(\delta_{\alpha}^{i}-\delta_{3}^{i} \frac{\partial_{\alpha}}{\partial_{3}}\right) \frac{\mathcal{A}_{i}}{\sqrt{4 \pi}} \\
& \tilde{\pi}_{a i}=\frac{\partial F}{\partial x_{a}^{i}}=p_{a i}-e_{a} \partial_{i} Q\left(\mathbf{x}_{a}\right) .
\end{aligned}
$$

The transverse part of $\mathcal{A}_{i}$, is connected with the new canonical variables as

$$
\stackrel{\perp}{\mathcal{A}_{i}}=\sqrt{4 \pi}\left(\delta_{i}^{\alpha}-\partial_{i} \Delta^{-1} \partial^{\alpha}\right) a_{\alpha}, \quad \alpha=1,2
$$

Now it is convenient to take $\mathbf{n}=(0,0,1)$ and perform the following canonical transformation of the field and particle momenta:

$$
\tilde{e}_{\alpha}=e_{\alpha}+\sqrt{4 \pi} \partial_{\alpha} \Delta^{-1} \rho, \quad \tilde{\pi}_{a i}=\pi_{a i}-\sqrt{4 \pi} e_{a} \partial_{i} \Delta^{-1} \partial^{\alpha} a_{\alpha}\left(\mathbf{x}_{a}\right) .
$$

The set of constraints for our system in the terms of the new variables can be written as follows:

$$
\left(\mathcal{E}, \Gamma, e_{\alpha}-a_{\alpha, 3}\right) \approx 0 .
$$

We reduce the second class constraints by means of the Dirac bracket:

$$
\begin{aligned}
\{F, G\}_{\mathrm{D}}=\{F, G\} & -\int \mathrm{d}^{3} x \mathrm{~d}^{3} y\left\{F, e_{\alpha}(t, \mathbf{x})-a_{\alpha, 3}(t, \mathbf{x})\right\} \\
& \times C^{\alpha \beta}(\mathbf{x}-\mathbf{y})\left\{e_{\beta}(t, \mathbf{y})-a_{\beta, 3}(t, \mathbf{y}), G\right\} .
\end{aligned}
$$

Here $\left\|C^{\alpha \beta}(\mathbf{x}-\mathbf{y})\right\|$ is an inverse matrix to

$$
\begin{gathered}
\mathbb{W}=\left\|\left\{\varepsilon_{\varepsilon}(t, \mathbf{x})-a_{\alpha, 3}(t, \mathbf{x}), e_{\beta}(t, \mathbf{y})-a_{\beta, 3}(t, \mathbf{y})\right\}\right\| \\
=\left\|-2 \delta_{\alpha \beta} \frac{\partial}{\partial x^{3}} \delta^{3}(\mathbf{x}-\mathbf{y})\right\| \\
\int C^{\alpha \gamma}(\mathbf{x}-\mathbf{z}) W_{\gamma \beta}(\mathbf{z}-\mathbf{y}) \mathrm{d}^{3} z=\delta_{\beta}^{\alpha} \delta^{3}(\mathbf{x}-\mathbf{y}) .
\end{gathered}
$$

It is given by

$$
C^{\alpha \beta}(\mathbf{x}-\mathbf{y})=-\delta^{\alpha \beta} \delta\left(x^{1}-y^{1}\right) \delta\left(x^{2}-y^{2}\right) \operatorname{sgn}\left(x^{3}-y^{3}\right),
$$

because $\left\|C^{\alpha \beta}(\mathbf{x}-\mathbf{y})\right\|$ must be antisymmetric.

Let us introduce the following denotation:

$$
\delta\left(x^{1}\right) \delta\left(x^{2}\right) \operatorname{sgn}\left(x^{3}\right) \equiv \frac{1}{2} \frac{1}{\partial_{3}} \delta^{3}(\mathbf{x}) .
$$

The final form of the Dirac bracket is

$$
\begin{aligned}
\{F, G\}_{\mathrm{D}}= & \{F, G\}_{\left(x_{a}, \pi_{a}\right)}+\{F, G\}_{\left(A_{0}, \mathcal{E}\right)}+\{F, G\}_{(Q, \Gamma)}+\frac{1}{2}\{F, G\}_{(a, e)} \\
& +\frac{1}{2} \int \mathrm{d}^{3} x\left[\frac{\delta F}{\delta e_{\alpha}(\mathbf{x})} \partial_{3} \frac{\delta G}{\delta e_{\alpha}(\mathbf{x})}-\frac{\delta F}{\delta a_{\alpha}(\mathbf{x})} \frac{1}{\partial_{3}} \frac{\delta F}{\delta a_{\alpha}(\mathbf{x})}\right]
\end{aligned}
$$


where $\{F, G\}_{(x, \pi)}$ denote the standard Poisson bracket in the terms of $x$ and $\pi$.

Elimination of constraints $(4.10)$ into $H_{\mathrm{c}}$ leads to the physical Hamiltonian:

$$
\begin{aligned}
H_{\mathrm{ph}}= & -\frac{1}{2} \sum_{a=1}^{N}\left[\pi_{a 3}+\frac{\left(\pi_{a \alpha}-\sqrt{4 \pi} e_{a} a_{\alpha}\left(\mathbf{x}_{a}\right)\right)^{2}+m_{a}^{2}}{\pi_{a 3}}\right]+\frac{1}{2} \sum_{a, b=1}^{N} \frac{e_{a} e_{b}}{\left|\mathbf{x}_{a}-\mathbf{x}_{b}\right|} \\
& -\frac{1}{2} \int\left(a_{\alpha}-\sqrt{4 \pi} \frac{\partial_{\alpha}}{\partial_{3}} \Delta^{-1} \rho\right) \Delta\left(a_{\alpha}-\sqrt{4 \pi} \frac{\partial_{\alpha}}{\partial_{3}} \Delta^{-1} \rho\right) \mathrm{d}^{3} x
\end{aligned}
$$

which generates the evolution of an arbitrary function $f$ depending on the gaugeinvariant variables $x_{a}^{i}, \pi_{a i}$ and $a_{\alpha}, e_{\alpha}$ in the terms of the Dirac bracket (4.16):

$$
\frac{\mathrm{d} f}{\mathrm{~d} t}=\frac{\partial f}{\partial t}+\left\{f, H_{\mathrm{ph}}\right\}_{\mathrm{D}}
$$

The gauge-invariant volume element of the constrained field phase space $\left(a_{\alpha}, e^{\alpha}\right)$ is written as

$$
\mathrm{d} \Gamma_{\mathrm{ph}}^{\mathrm{f}}(t)=\gamma \sqrt{\operatorname{Det} \mathbb{W}} \prod_{\alpha=1,2} \prod_{\mathbf{x}} \delta\left[e_{\alpha}(t, \mathbf{x})-a_{\alpha, 3}(t, \mathbf{x})\right] \mathrm{d} a_{\alpha}(t, \mathbf{x}) \mathrm{d} e_{\alpha}(t, \mathbf{x}),
$$

where $\gamma$ is defined as a normalization constant of the Gauss integral [7]:

$$
\begin{aligned}
& \gamma \int \exp \left(-\frac{1}{2} \int a_{\alpha}(t, \mathbf{x}) L^{\alpha \beta}(t, \mathbf{x}, \mathbf{y}) a_{\beta}(t, \mathbf{y}) \mathrm{d}^{3} x \mathrm{~d}^{3} y\right) \prod_{\alpha=1,2} \prod_{\mathbf{x}} \mathrm{d} a_{\alpha}(t, \mathbf{x}) \\
& =\operatorname{Det}^{-1 / 2}\left\|L^{\alpha \beta}(t, \mathbf{x}, \mathbf{y})\right\| .
\end{aligned}
$$

Taking into account (4.19), we can write the volume element of the physical phase space of the described system:

$$
\mathrm{d} \Gamma_{\mathrm{ph}}(t)=\mathrm{d} \Gamma_{\mathrm{ph}}^{\mathrm{p}}(t) \mathrm{d} \Gamma_{\mathrm{ph}}^{\mathrm{f}}(t) .
$$

Here

$$
\mathrm{d} \Gamma_{\mathrm{ph}}^{\mathrm{p}}(t)=\prod_{a=1}^{N} \prod_{i=1}^{3} \mathrm{~d} x_{a}^{i}(t) \mathrm{d} \pi_{i a}(t) .
$$

is the volume element of the particle phase space.

Now we need check the Liouville theorem: $\Gamma_{\mathrm{ph}}(t)=\Gamma_{\mathrm{ph}}\left(t_{0}\right)$.

It is well known from the classical mechanics, that system evolution in the phase space can be described by means of a canonical transformation in the terms of the Poisson bracket, which immediately leads to the conservation of the phase space volume. This proves the conservation of $\Gamma_{\mathrm{ph}}^{\mathrm{p}}$. In our case, the field evolution is generated by the Dirac bracket. Nevertheless, the conservation of the volume of the constrained field phase space can be proved and we shall demonstrate its possible evidence for the considered Dirac bracket (4.16) in Appendix B.

The volume element of the full phase space is

$$
\mathrm{d} \Gamma_{\text {full }}(t)=\mathrm{d} \Gamma_{\mathrm{ph}}(t) \mathrm{d} \Gamma_{\mathrm{g}}(t),
$$


where $\mathrm{d} \Gamma_{\mathrm{g}}(t)$ is the gauge volume element of the phase space:

$$
\prod_{\mathbf{x}} \delta[\mathcal{E}(t, \mathbf{x})] \delta[\Gamma(t, \mathbf{x})] \mathrm{d} A_{0}(t, \mathbf{x}) \mathrm{d} \mathcal{E}(t, \mathbf{x}) \mathrm{d} Q(t, \mathbf{x}) \mathrm{d} \Gamma(t, \mathbf{x}) .
$$

However, we eliminate $\mathrm{d} \Gamma_{\mathrm{g}}(t)$ from the further description, because all thermodynamical characteristics of the system do not depend on dynamics of the gauge degrees of freedom (see [15]).

\section{Statistical mechanics}

Now let us imagine that the physical initial data $x_{a}^{i}\left(t_{0}\right), \pi_{a i}\left(t_{0}\right), a_{\alpha}\left(t_{0}, \mathbf{x}\right), e_{\alpha}\left(t_{0}, \mathbf{x}\right)$ are not precisely known. Hence, we can introduce a probability density $\varrho\left(t_{0}\right) \equiv$ $\varrho\left(t_{0}, x_{a}^{i}\left(t_{0}\right), \pi_{a i}\left(t_{0}\right), a_{\alpha}\left(t_{0}, \mathbf{x}\right), e_{\alpha}\left(t_{0}, \mathbf{x}\right)\right)$ for having various initial states. This function satisfies the condition

$$
\int \varrho\left(t_{0}\right) \mathrm{d} \Gamma_{\mathrm{ph}}\left(t_{0}\right)=1
$$

Then the average value of a general dynamical variable $f$ is defined by

$$
\bar{f}(t)=\int f\left(t, x_{a}^{i}(t), \pi_{a i}(t), a_{\alpha}(t, \mathbf{x}), e_{\alpha}(t, \mathbf{x})\right) \varrho(t) \mathrm{d} \Gamma_{\mathrm{ph}}(t) .
$$

Since $t_{0}$ has been randomly selected, we come to the relation

$$
1=\int \varrho\left(t_{0}\right) \mathrm{d} \Gamma_{\mathrm{ph}}\left(t_{0}\right)=\int \varrho(t) \mathrm{d} \Gamma_{\mathrm{ph}}(t)
$$

We have already seen that $\mathrm{d} \Gamma_{\mathrm{ph}}\left(t_{0}\right)=\mathrm{d} \Gamma_{\mathrm{ph}}(t)$, so one immediately obtains the Liouville equation

$$
0=\frac{\mathrm{d} \varrho(t)}{\mathrm{d} t}=\frac{\partial \varrho(t)}{\partial t}+\left\{\varrho(t), H_{\mathrm{ph}}(t)\right\}_{\mathrm{D}}
$$

Taking into account (5.4), we interpret $\varrho$ as an integral of motion.

Let us consider the canonical Gibbs ensemble in equilibrium. In this case we have

$$
\varrho(t)=C \mathrm{e}^{-\beta H_{\mathrm{ph}}(t)},
$$

where $\beta=1 / k T$ and $C$ is a normalization constant.

Partition function can be found as

$$
Z=\int \frac{\mathrm{e}^{-\beta H_{\mathrm{ph}}(t)}}{(2 \pi)^{3 N} N !} \mathrm{d} \Gamma_{\mathrm{ph}}(t) .
$$

We shall find the value of $Z$ below.

Let us first rewrite the physical Hamiltonian as follows

$$
H_{\mathrm{ph}}=-\frac{1}{2} \sum_{a=1}^{N}\left(\pi_{a 3}+\frac{\pi_{a \alpha}^{2}+m_{a}^{2}}{\pi_{a 3}}\right)+\frac{1}{2} \sum_{a, b=1}^{N} \frac{e_{a} e_{b}}{\left|\mathbf{x}_{a}-\mathbf{x}_{b}\right|}
$$




$$
\begin{aligned}
& -2 \pi \int \frac{\partial_{\alpha}}{\partial_{3}} \rho(t, \mathbf{x}) \Delta^{-1}(\mathbf{x}-\mathbf{y}) \frac{\partial_{\alpha}}{\partial_{3}} \rho(t, \mathbf{y}) \mathrm{d}^{3} x \mathrm{~d}^{3} y \\
& +2 \pi \int\left(\frac{\partial_{\alpha}}{\partial_{3}} \rho(t, \mathbf{x})+j_{\alpha}^{0}(t, \mathbf{x})\right) G(t, \mathbf{x}-\mathbf{y})\left(\frac{\partial_{\alpha}}{\partial_{3}} \rho(t, \mathbf{y})+j_{\alpha}^{0}(t, \mathbf{y})\right) \mathrm{d}^{3} x \mathrm{~d}^{3} y \\
& -\frac{1}{2} \int \tilde{a}_{\alpha}(t, \mathbf{x})\left(\Delta+4 \pi \sum_{a=1}^{N} \frac{e_{a}^{2}}{\pi_{a 3}(t)} \delta^{3}\left(\mathbf{x}-\mathbf{x}_{a}(t)\right)\right) \tilde{a}_{\alpha}(t, \mathbf{x}) \mathrm{d}^{3} x
\end{aligned}
$$

where

$$
\begin{aligned}
& \tilde{a}_{\alpha}(t, \mathbf{x})=a_{\alpha}(t, \mathbf{x})-\sqrt{4 \pi} \int G(t, \mathbf{x}-\mathbf{y})\left(\frac{\partial_{\alpha}}{\partial_{3}} \rho(t, \mathbf{y})+j_{\alpha}^{0}(t, \mathbf{y})\right) \mathrm{d}^{3} y \\
& j_{\alpha}^{0}(t, \mathbf{x})=-\sum_{a=1}^{N} e_{a} \frac{\pi_{a \alpha}(t)}{\pi_{a 3}(t)} \delta^{3}\left(\mathbf{x}-\mathbf{x}_{a}(t)\right) \\
& {\left[\Delta+4 \pi \sum_{a=1}^{N} \frac{e_{a}^{2}}{\pi_{a 3}(t)} \delta^{3}\left(\mathbf{x}-\mathbf{x}_{a}(t)\right)\right] G(t, \mathbf{x})=\delta^{3}(\mathbf{x}) .}
\end{aligned}
$$

Since $H_{\mathrm{ph}}$ does not depend on field momenta $e^{\alpha}$, after an integration over $e^{\alpha}$ in (5.6) we obtain the expression for the partition function:

$$
\begin{aligned}
Z= & \gamma \sqrt{\operatorname{Det} \mathbb{W}} \frac{1}{N !} \int \exp (-\beta)\left[-\frac{1}{2} \sum_{a=1}^{N}\left(\pi_{a 3}+\frac{\pi_{a \alpha}^{2}+m_{a}^{2}}{\pi_{a 3}}\right)+\frac{1}{2} \sum_{a, b=1}^{N} \frac{e_{a} e_{b}}{\left|\mathbf{x}_{a}-\mathbf{x}_{b}\right|}\right. \\
& -2 \pi \int \frac{\partial_{\alpha}}{\partial_{3}} \rho(t, \mathbf{x}) \Delta^{-1}(\mathbf{x}-\mathbf{y}) \frac{\partial_{\alpha}}{\partial_{3}} \rho(t, \mathbf{y}) \mathrm{d}^{3} x \mathrm{~d}^{3} y \\
& +2 \pi \int\left(\frac{\partial_{\alpha}}{\partial_{3}} \rho(t, \mathbf{x})+j_{\alpha}^{0}(t, \mathbf{x})\right) G(t, \mathbf{x}-\mathbf{y})\left(\frac{\partial_{\alpha}}{\partial_{3}} \rho(t, \mathbf{y})+j_{\alpha}^{0}(t, \mathbf{y})\right) \mathrm{d}^{3} x \mathrm{~d}^{3} y \\
& \left.-\frac{1}{2} \int \tilde{a}_{\alpha}(t, \mathbf{x})\left(\Delta+4 \pi \sum_{a=1}^{N} \frac{e_{a}^{2}}{\pi_{a 3}(t)} \delta^{3}\left(\mathbf{x}-\mathbf{x}_{a}(t)\right)\right) \tilde{a}_{\alpha}(t, \mathbf{x}) \mathrm{d}^{3} x\right] \\
& \times \prod_{a=1}^{N} \prod_{i=1}^{3} \frac{\mathrm{d} x_{a}^{i} \mathrm{~d} \pi_{a i}}{2 \pi} \prod_{\alpha=1,2} \prod_{\mathbf{x}} \mathrm{d} a_{\alpha}(t, \mathbf{x})
\end{aligned}
$$

Here we can replace $\mathrm{d} a_{\alpha}$ by $\mathrm{d} \tilde{a}_{\alpha}$. Taking into account (4.8), the integration over $\tilde{a}_{\alpha}$ yields

$$
\begin{aligned}
Z= & Z^{\mathrm{f}} \frac{1}{N !} \int \prod_{a=1}^{N} \prod_{i=1}^{3} \frac{\mathrm{d} x_{a}^{i} \mathrm{~d} \pi_{a i}}{2 \pi} \exp (-\beta)\left[-\frac{1}{2} \sum_{a=1}^{N}\left(\pi_{a 3}+\frac{\pi_{a \alpha}^{2}+m_{a}^{2}}{\pi_{a 3}}\right)\right. \\
& +\frac{1}{2} \sum_{a, b=1}^{N} \frac{e_{a} e_{b}}{\left|\mathbf{x}_{a}-\mathbf{x}_{b}\right|}-2 \pi \int \frac{\partial_{\alpha}}{\partial_{3}} \rho(t, \mathbf{x}) \Delta^{-1}(\mathbf{x}-\mathbf{y}) \frac{\partial_{\alpha}}{\partial_{3}} \rho(t, \mathbf{y}) \mathrm{d}^{3} x \mathrm{~d}^{3} y \\
& \left.+2 \pi \int\left(\frac{\partial_{\alpha}}{\partial_{3}} \rho(t, \mathbf{x})+j_{\alpha}^{0}(t, \mathbf{x})\right) G(t, \mathbf{x}-\mathbf{y})\left(\frac{\partial_{\alpha}}{\partial_{3}} \rho(t, \mathbf{y})+j_{\alpha}^{0}(t, \mathbf{y})\right) \mathrm{d}^{3} x \mathrm{~d}^{3} y\right] \\
& \times \operatorname{Det}^{-1 / 2}\left\|\delta^{\alpha \beta}\left(\delta^{3}(\mathbf{x}-\mathbf{y})+4 \pi \Delta^{-1}(\mathbf{x}-\mathbf{y}) \sum_{a=1}^{N} \frac{e_{a}^{2}}{\pi_{a 3}} \delta^{3}\left(\mathbf{y}-\mathbf{x}_{a}\right)\right)\right\|,
\end{aligned}
$$


where

$$
Z^{\mathrm{f}}=\frac{\sqrt{\operatorname{Det} \mathbb{W}}}{\sqrt{\operatorname{Det}\left\|-\beta \delta^{\alpha \beta} \Delta \delta^{3}(\mathbf{x}-\mathbf{y})\right\|}}
$$

represents the free field partition function.

\section{Conclusions}

In this paper we have once more demonstrated the usefulness of the various forms of relativistic dynamics in treating the relativistic particle systems. Constraint analysis of the considered problem shows significantly different structures of Hamiltonian description of charged particles, interacting by means of an electromagnetic field, in the space-like and isotropic forms of dynamics. Specifically, the use of the front form of relativistic dynamics allows us to exclude the electromagnetic field variables from the classical partition function of the system of charged particles. It is obvious that such an exclusion must depend on the particular boundary conditions for the field variables, but at present the proper sense of these conditions remains unclear. The obtained representation for the partition function contains highly nonlocal expressions, and their further analysis consists of a complicated task. The consideration of the various approximation schemes at this step seems to be inevitable.

It should be noted that the exclusion of the field variables transform the problem into the domain of relativistic direct interaction theory (see, e.g., [24,25]). An application of such a theory to the consistent formulation of the relativistic statistical mechanics is just at the beginning.

On the other hand, the established Liouville equation may be used in nonequilibrium situation as well. It serves an useful starting point for deriving various new forms of kinetic equations for a charged particle system.

\section{Acknowledgement}

We are greatly indebted to Yu.Yaremko and V.Shpytko for many stimulating discussions. Numerous helpful conversations with A.Duviryak and his support in the performance of this work are especially acknowledged.

\section{A. Relativistic free particle in an arbitrary form of dynamics}

The Lagrangian of relativistic free particle in a given form of dynamics (2.5) is

$$
L=-m \sqrt{(D \varphi)^{2}-\mathbf{v}^{2}} \equiv-m \Gamma^{-1} .
$$

The canonical momentum and Hamiltonian are given by

$$
\begin{gathered}
p_{i}=m \Gamma\left(g_{i j} v^{j}-\varphi_{i} \varphi_{t}\right), \\
H=m \Gamma \varphi_{t} D \varphi,
\end{gathered}
$$


where the matrix $g_{i j}=\delta_{i j}-\varphi_{i} \varphi_{j}$ has been introduced. The determinant of the matrix is

$$
g \equiv\left\|g_{i j}\right\|=1-\varphi^{2}
$$

and $g \geqslant 0$ as the result of the condition (2.10).

Consider firstly the case $g>0$. Then the matrix $g_{i j}$ has an inverse, $\tilde{g}_{i j}=\delta_{i j}+$ $g^{-1} \varphi_{i} \varphi_{j}$ and from (A.2) it follows

$$
\tilde{g}_{i j} p_{i} p_{j}=m^{2}\left(\Gamma^{2} g^{-1} \varphi_{t}^{2}-1\right) .
$$

Using (2.8), we find

$$
\begin{gathered}
m \Gamma=\varphi_{t}^{-1} \sqrt{g\left(m^{2}+\mathbf{p}^{2}\right)+\left(p_{i} \varphi_{i}\right)} \equiv \varphi_{t}^{-1} B, \\
v_{i}=B^{-1} \varphi_{t}\left[p_{i}+g^{-1} \varphi_{i}\left(B+p_{j} \varphi^{j}\right)\right] \\
D \varphi=B^{-1} \varphi_{t} g^{-1}\left(B+p_{i} \varphi^{i}\right)
\end{gathered}
$$

Combining these results with (A.3), one gets

$$
H=\varphi_{t} g^{-1}\left[\sqrt{g\left(m^{2}+\mathbf{p}^{2}\right)+\left(p_{i} \varphi^{i}\right)^{2}}+p_{i} \varphi^{i}\right] .
$$

The case of isotropic forms of mechanics can be treated by taking the limit $g \rightarrow+0$ and using that, in view of (A.2),

$$
p_{i} \varphi^{i}=m \Gamma\left[g v_{i} \varphi^{i}+\varphi_{t}(g-1)\right],
$$

so that $p_{i} \varphi^{i} \leqslant 0$ as $g \rightarrow+0$. It gives

$$
H=-\frac{1}{2} \varphi_{t} \frac{m^{2}+\mathbf{p}^{2}}{p_{i} \varphi^{i}}
$$

\section{B. Proof of the Liouville theorem for a given Dirac bracket}

Let us show that the evolution of the field variables as the generalized canonical transformation in the terms of the Dirac bracket [2] conserves the volume of the constrained field phase space.

We first consider the transformation generated by some functional $G$ with an arbitrary parameter $\xi$ :

$$
\begin{aligned}
& \alpha_{\alpha}=a_{\alpha}+\xi\left\{a_{\alpha}, G\right\}_{\mathrm{D}}=a_{\alpha}+\frac{\xi}{2} \frac{\delta G}{\delta e^{\alpha}}-\frac{\xi}{2} \frac{1}{\partial_{3}} \frac{\delta G}{\delta a^{\alpha}} \\
& \epsilon_{\alpha}=e_{\alpha}+\xi\left\{e_{\alpha}, G\right\}_{\mathrm{D}}=e_{\alpha}-\frac{\xi}{2} \frac{\delta G}{\delta a^{\alpha}}+\frac{\xi}{2} \partial_{3} \frac{\delta G}{\delta e^{\alpha}} .
\end{aligned}
$$

We immediately see that $e_{\alpha}-a_{\alpha, 3}=\epsilon_{\alpha}-\alpha_{\alpha, 3}$. So, the constancy condition of $\Gamma_{\mathrm{ph}}^{\mathrm{f}}$ leads to the relation:

$$
\begin{aligned}
\int & \prod_{\alpha=1,2} \prod_{\mathbf{x}} \delta\left[\epsilon_{\alpha}(t, \mathbf{x})-\alpha_{\alpha, 3}(t, \mathbf{x})\right] \mathrm{d} \alpha_{\alpha}(t, \mathbf{x}) \mathrm{d} \epsilon_{\alpha}(t, \mathbf{x})= \\
= & \int J(\xi, 0) \prod_{\alpha=1,2} \prod_{\mathbf{x}} \delta\left[e_{\alpha}(t, \mathbf{x})-a_{\alpha, 3}(t, \mathbf{x})\right] \mathrm{d} a_{\alpha}(t, \mathbf{x}) \mathrm{d} e_{\alpha}(t, \mathbf{x}) .
\end{aligned}
$$


Here Jacobian $J(\xi, \eta)$ is defined by

$$
J(\xi, \eta) \equiv \operatorname{Det}\left\|\frac{\delta\left(\alpha_{\alpha}(t, \mathbf{x} ; \xi) \epsilon_{\alpha}(t, \mathbf{x} ; \xi)\right.}{\delta\left(\alpha_{\beta}(t, \mathbf{y} ; \eta) \epsilon_{\beta}(t, \mathbf{y} ; \eta)\right.}\right\| .
$$

If $\eta=0$, then we have

$$
J(\xi, 0)=\operatorname{Det}\left\|\frac{\delta\left(\alpha_{\alpha}(t, \mathbf{x} ; \xi) \epsilon_{\alpha}(t, \mathbf{x} ; \xi)\right.}{\delta\left(a_{\beta}(t, \mathbf{y}) e_{\beta}(t, \mathbf{y})\right)}\right\|
$$

It is evident that $J(0,0)=1$. Let us compute $\left.(\mathrm{d} J(\xi, 0) / \mathrm{d} \xi)\right|_{\xi=0}$. We get

$$
\begin{aligned}
\left.\frac{\mathrm{d} J(\xi, 0)}{\mathrm{d} \xi}\right|_{\xi=0}= & \left.\operatorname{Tr}\left\|\frac{\delta \partial_{\xi} \alpha_{\alpha}(t, \mathbf{x} ; \xi)}{\delta a_{\beta}(t, \mathbf{y})}\right\|\right|_{\xi=0}+\operatorname{Tr}\left\|\frac{\delta \partial_{\xi} \epsilon_{\alpha}(t, \mathbf{x} ; \xi)}{\delta e_{\beta}(t, \mathbf{y})}\right\| \|_{\xi=0} \\
= & \operatorname{Tr}\left\|\frac{1}{2} \frac{\delta^{2} G}{\delta a_{\beta}(t, \mathbf{y}) \delta e_{\alpha}(t, \mathbf{x})}\right\|+\operatorname{Tr}\left\|-\frac{1}{2} \frac{1}{\partial_{3}} \frac{\delta^{2} G}{\delta a_{\beta}(t, \mathbf{y}) \delta a_{\alpha}(t, \mathbf{x})}\right\| \\
& +\operatorname{Tr}\left\|-\frac{1}{2} \frac{\delta^{2} G}{\delta e_{\beta}(t, \mathbf{y}) \delta a_{\alpha}(t, \mathbf{x})}\right\|+\operatorname{Tr}\left\|\frac{1}{2} \partial_{3} \frac{\delta^{2} G}{\delta e_{\beta}(t, \mathbf{y}) \delta e_{\alpha}(t, \mathbf{x})}\right\| .
\end{aligned}
$$

It is obvious that the first and the third terms vanish. One of the possible ways demonstrating a cancellation of the second and the fourth terms is based on the commutation of the Dirac bracket action and the replacement of $a_{\alpha, 3}$ by $e_{\alpha}$ (or $e_{\alpha}$ by $a_{\alpha, 3}$ in view of $\left.e_{\alpha}-a_{\alpha, 3}=0\right)$.

Therefore, we have

$$
\left.\frac{\mathrm{d} J(\xi, 0)}{\mathrm{d} \xi}\right|_{\xi=0}=0
$$

Since $J(\xi, 0)=J\left(\xi, \xi_{1}\right) J\left(\xi_{1}, 0\right)$, then

$$
\left.\frac{\mathrm{d} J(\xi, 0)}{\mathrm{d} \xi}\right|_{\xi=\xi_{1}}=\left.\frac{\mathrm{d} J\left(\xi, \xi_{1}\right)}{\mathrm{d} \xi}\right|_{\xi=\xi_{1}} J\left(\xi_{1}, 0\right)=0 .
$$

Thus, $J(\xi, 0)=1$ for all $\xi$.

Now if we take $\xi=t$ and $G=H_{\mathrm{ph}}$, we come to a conclusion

$$
\Gamma_{\mathrm{ph}}(t)=\Gamma_{\mathrm{ph}}\left(t_{0}\right)
$$

namely, the time evolution preserves the phase space volume $\Gamma_{\mathrm{ph}}$.

\section{References}

1. Dirac P.A.M. Lectures on Quantum Mechanics. New York, Yeshiva Univ., 1964.

2. Sudarshan E.C.G., Mukunda N. Classical Dynamics: A Modern Perspective. New York, Wiley, 1974.

3. Sundermeyer K. Constrained Dynamics. (Lecture Notes in Physics, vol. 169). New York, Springer-Verlag, 1982. 
4. Marmo G., Mendella G., Tulczyew W.M. Constrained Hamiltonian systems as implicit differential equations. // J. Phys. A: Math. Gen., 1997, vol. 30, p. 277-293.

5. Gotay M.J., Isenberg J., Marsden J.E. Momentum map and classical relativistic fields. Part I. Preprint physics/98010119, 1998.

6. Fadeev L.D. Feynman integral for singular Lagrangians. // Theor. Math. Phys., 1969, vol. 1, No. 1, p. 1.

7. Slavnov A.A., Faddeev L.D. An Introduction to Quantum Theory of Gauge Fields. Moscow, Nauka, 1978 (in Russian).

8. Horwitz L.P., Sciewe W.C., Piron C. Gibbs ensembles in relativistic classical and quantum mechanics. // Ann. of Phys., 1981, vol. 137, p. 306-340.

9. Pavlotsky I.P. Foundations of weak relativistic mechanics. Moscow, High School, 1983 (in Russian).

10. Umezawa H., Matsumoto H., Tachiki M. Thermo Field Dynamics and Condensed States. Amsterdam, North Holland, 1982.

11. Miller D.E., Karsch F. Covariant structure of relativistic gases in equilibrium. // Phys. Rev. D, 1981, vol. 24, No. 10, p. 2564-2575.

12. Dirac P.A.M. The Hamiltonian form of field dynamics. // Can. J. Math., 1951, vol. 3, No. 1, p. 1-23.

13. Dirac P.A.M. Forms of relativistic dynamics. // Rev. Mod. Phys., 1949, vol. 21, No. 3, p. 392-399.

14. Lusanna L. The $N$ - and 1-time classical descriptions of $N$-body relativistic kinematics and the electromagnetic interaction. // Int. J. Mod. Phys., 1997, vol. 12, No. 4, p. 645722 .

15. Duviryak A., Nazarenko A. The Liouville equation for systems with constraints. // J. Phys. Stud. (in press).

16. Landau L.D., Lifshitz E.M. Field Theory. Nauka, Moscow, 1973 (in Russian).

17. Rohrlich F. Classical Charged Particles: Foundations of Their Theory. AddisonWesley, New York, 1990.

18. Parrott S. Relativistic Electrodynamics and Differential Geometry. Springer, New York, 1987.

19. Gaida R.P., Kluchkovsky Yu.B., Tretyak V.I. Forms of relativistic dynamics in the classical Lagrangian description of a system of particles. // Teor. Mat. Fiz., 1983, vol. 55, No. 1, p. 88-105 (in Russian; transl. in English: Theor. Math. Phys., 1983, vol. 55 , No. 1 , p. $372-384)$.

20. Gaida R.P., Kluchkovsky Yu.B., Tretyak V.I. Three-dimensional Lagrangian approach to the classical relativistic dynamics of directly interacting particles. - In: Constraint's Theory and Relativistic Dynamics. Longhi G. and Lusanna L., eds. Singapore, World Scientific Publ., 1987, p. 210-241.

21. Duviryak A., Shpytko V., Tretyak V. Isotropic forms of dynamics in the relativistic direct interaction theory. // Cond. Matter Phys., 1998, vol. 1, No. 3(15), p. 463-512.

22. Tretyak V.I. Classical Lagrangian description of the particle system in various forms of relativistic dynamics. Preprint of the Institute for Theoretical Physics, ITP-82-87, Kiev, 1982 (in Russian).

23. Gitman D.M., Tyutin I.V. Canonical quantization of the fields with constraints. Nauka, Moscow, 1986 (in Russian)

24. Hoyle F., Narlikar J.V. Action-at-a-Distance in Physics and Cosmology. Freeman, San Francisco, 1974. 
25. Gaida R.P. Quasirelativistic systems of interacting particles. // Sov. J. Part. Nucl., 1982, vol. 13, p. 179.

\title{
Класичні релятивістичні системи заряджених частинок у фронтовій формі динаміки та рівняння Ліувіля
}

\author{
А.Назаренко, В.Третяк \\ Інститут фізики конденсованих систем НАН України, \\ 79011 Львів, вул. Свєнціцького, 1 \\ Отримано 20 грудня 1999 р.
}

Розглянуто класичну релятивістичну систему точкових частинок у взаємодії з електромагнітним полем у тривимірному зображенні. За допомогою геометричної концепції форм релятивістичної динаміки виключається калібрувальна свобода, пов'язана з хронометричною інваріантністю чотиривимірного опису. Решта калібрувальних ступенів вільности електромагнітного потенціалу аналізується в рамках гамільтонової механіки з в' язями Дірака у фронтовій формі динаміки. Одержані результати застосовано до проблем релятивістичної статистичної механіки. На основі відповідного рівняння Ліувіля записано статистичну суму системи у калібрувально-інваріантний спосіб та здійснено інтеґрування за польовими змінними.

Ключові слова: класична релятивістична механіка, форми релятивістичної динаміки, релятивістична статистична механіка, заряджені частинки, рівняння Ліувіля

PACS: 03.30. $+p, 05.20 .-y$ 